\title{
Fibrolamellar hepatocellular carcinoma: current clinical perspectives
}

\author{
This article was published in the following Dove Press journal: \\ Journal of Hepatocellular Carcinoma \\ 9 October 2015 \\ Number of times this article has been viewed
}

\author{
Kelly J Lafaro' \\ Timothy M Pawlik ${ }^{2}$ \\ 'Center for Pancreatic Cancer \\ Research, Memorial Sloan Kettering \\ Cancer Center, New York, NY, \\ ${ }^{2}$ Department of Surgery, The Johns \\ Hopkins University School of \\ Medicine, Baltimore, MD, USA
}

Correspondence: Timothy M Pawlik Division of Surgical Oncology, Department of Surgery, Johns Hopkins Hospital, 600 North Wolfe Street, Blalock 688, Baltimore, MD 21287 , USA $\mathrm{Tel}+$ I 4105022387

Fax + I 4105022388

Email tpawlik।@jhmi.edu
Abstract: Fibrolamellar carcinoma (FLC) is a variant of hepatocellular carcinoma (HCC), which comprises $\sim 1 \%-9 \%$ of all HCCs. Although FLC is a variant of HCC, it is distinct from $\mathrm{HCC}$ in that it most often affects younger patients (10-35 years of age) with no underlying liver disease. FLC often presents with vague abdominal pain, nausea, abdominal fullness, malaise, and weight loss. Surgery is the current mainstay of treatment for FLC and remains the only potentially curative option. While FLCs are considered less responsive to chemotherapy than their classic HCC counterparts, there have been suggestions that multimodality treatments may be effective, especially in advanced cases. Further research is necessary to determine effective systemic therapies as an adjunct to surgery for FLC.

Keywords: hepatocellular carcinoma, fibrolamellar, hepatocyte paraffin I, locoregional therapy

\section{Introduction}

Fibrolamellar carcinoma (FLC) is a variant of hepatocellular carcinoma (HCC) that comprises $\sim 1 \%-9 \%$ of all HCCs according to the SEER database. ${ }^{1}$ FLC was first described by Edmondson in 1956 as an adult type of liver tumor in a 14-year-old female with no underlying liver disease. ${ }^{2}$ The term FLC was not suggested, however, until 1980 when Craig et al described a set of patients with a unique histologic variant of HCC. ${ }^{3}$ The World Health Organization (WHO) Classification of Tumors subsequently recognized FLC as having a unique histological pattern; however, it took until 2010 for the WHO to designate this clinical entity with its own WHO classification number. ${ }^{4}$

Although FLC is a variant of HCC, it is distinct from HCC in that FLC most often affects younger patients (10-35 years of age) with no underlying liver disease. . $^{5,6}$ On pathological analysis, FLC is characterized by large tumor cells with deeply eosinophilic cytoplasm due to abundant mitochondria and prominent nuclei arranged in cords surrounded by lamellated collagen fibers. ${ }^{3,7}$ The tumor cells can demonstrate hepatocellular features; however, FLC tumors also can display both biliary and neuroendocrine differentiation. While the etiology of FLC tumors remains still unclear, FLC is thought to have an overall better prognosis than other primary liver tumors (eg, HCC, intrahepatic cholangiocarcinoma). We herein review the epidemiology, diagnosis, treatment, and prognosis of patients with FLC.

\section{Epidemiology}

FLC accounts for between $1 \%$ and $9 \%$ of all HCCs depending on the population studied. ${ }^{8-15}$ From an epidemiologic viewpoint, one feature that often distinguishes FLC 
from HCC is the age at diagnosis. FLC typically occurs in young adults, with most patients being 10-35 years of age at presentation compared with an average age of 65 years at presentation among patients with $\mathrm{HCC} .{ }^{5}$ Compared with HCC, some studies note that FLC patients are more likely to be female, while others have noted no specific sex predilection..$^{5,10,13}$ Regarding race, one study that utilized SEER data noted a higher incidence of FLC versus HCC among patients of Caucasian ethnicity; however, the association of race with HCC subtype did not remain significant after adjustment for age. ${ }^{5}$ In addition, FLC has been reported with similar prevalence in countries across the globe including the United States, Mexico, Sweden, Saudi Arabia, Thailand, France, Canada, South Africa, Japan, Korea, India, Taiwan, and United Kingdom. As such, the data would suggest that there is no strong association of race or ethnicity with the risk of FLC. ${ }^{5,8-26}$

\section{Presentation}

FLC often presents with vague abdominal pain, nausea, abdominal fullness, malaise, and weight loss. ${ }^{3}$ While the physical examination is often within normal limits, when present, common physical findings include a palpable abdominal mass or hepatomegaly with or without pain in the right upper quadrant. Other reported rare presentations include jaundice due to biliary obstruction, ${ }^{27-29}$ gynecomastia in males, ${ }^{30}$ fulminant liver failure, ${ }^{6,31-33}$ recurrent deep vein thrombosis, ${ }^{34}$ encephalopathy, ${ }^{35}$ thrombophlebitis of the lower extremity, ${ }^{36}$ anemia, ${ }^{37}$ ascites,${ }^{38}$ and hypoglycemia. ${ }^{39}$

On serum analysis, beta human chorionic gonadotropin can sometimes be elevated. ${ }^{40}$ Typically, liver function markers such as aspartate aminotransferase, alanine aminotransferase, and alkaline phosphatase levels are normal or mildly elevated. ${ }^{3,10,41}$ Elevated alkaline phosphatase levels in the setting of FLC are likely due to the growth of the tumor into the biliary tree or biliary obstruction. ${ }^{42}$ Alpha fetoprotein levels are predominately normal in patients with FLC unlike in traditional HCC. ${ }^{43}$ While uncommon, other serum proteins may be elevated with FLC. For example, transcobalamin I (also known as haptocortin), which normally protects vitamin B12 from degradation in the digestive tract, may be elevated. ${ }^{44-46}$ Transcobalamin II, a vitamin B12-binding protein induced by vitamin $\mathrm{K}$ absence/antagonist-II levels, may also be high. ${ }^{13,22,47}$ Less frequently, elevations in serum proteins including fibrinogen and neurotensin can be noted. ${ }^{33,48}$ No large study has determined, however, the diagnostic accuracy of these serum proteins as tumor markers.

\section{Diagnosis}

\section{Imaging}

Due to the vague symptoms associated with FLC, diagnosis is usually made on the basis of both clinical presentation and diagnostic imaging studies. Imaging studies including ultrasound, computed tomography (CT) scan, and magnetic resonance imaging (MRI) may all be useful. On ultrasound, FLC is characterized as a well-defined mass that has heterogenous echogenicity. ${ }^{49}$ Rather than ultrasound, cross-sectional imaging is the preferred mechanism to characterize most liver lesions, including FLC. CT scans that included an unenhanced phase followed by an intravenous contrast-enhanced hepatic arterial phase, a portal venous phase, and a delayed phase are recommended. ${ }^{50}$ Using contrast-enhanced CT, FLC typically presents as a large $(7-20 \mathrm{~cm})$, heterogeneous, welldefined mass with a lobulated outline. ${ }^{51}$ On the unenhanced phase, FLC is most often hypoattenuating with calcifications (40\%-68\%) and a central stellate scar (65\%-75\%), which is not seen in traditional HCC. ${ }^{49,51,52}$ Necrosis without intratumoral hemorrhage is also a common finding in FLC. ${ }^{49,51}$ On hepatic arterial phase, most FLC lesions appear with heterogeneous hyperattenuation due to the large hypervascular tumor cells surrounding hypovascular fibrotic bands, as well as necrosis. ${ }^{49,51}$ The portal venous-phase CT characteristics of FLC are more variable. In $\sim 50 \%$ of patients, FLC tumors are isoattenuating to the liver in the portal venous phase, while in $30 \%-40 \%$ and $10 \%-20 \%$, the lesions are either hyperattenuating or hypoattenuating, respectively. ${ }^{50,51}$

In many centers, MRI is the preferred imaging modality. MRI can be quite helpful in distinguishing FLC from other liver lesions. FLC tumors are usually hypointense on T1-weighted images and hyperintense on T2-weighted images with a fibrous central scar that remains hypointense on both T1- and T2-weighted images. ${ }^{50,51}$ The hypointensity of the central scar can help differentiate FLC from benign liver masses such as focal nodular hyperplasia, which typically has a predominately hyperintense central scar on T2-weighted images. Gadolinium contrast-enhanced MRI is used by many institutions to help further characterize liver lesions. On gadolinium-enhanced MRI, FLC is characterized by marked heterogeneous enhancement on the arterial phase that washes out and leaves an isointense or hypointense lesion on the portal venous phase..$^{50,51}$

The role of ${ }^{18} \mathrm{~F}$-FDG positron emission tomographycomputed tomography (PET/CT) in the workup of FLC has not been well studied. Limited case series have suggested that $\mathrm{PET} / \mathrm{CT}$ may be a useful tool in the diagnosis and monitoring of FLC as it may be FDG avid in up to $75 \%$ of patients 
(Figure 1). ${ }^{53,54}$ As such, FDG-PET may be especially helpful in distinguishing FLC from focal nodular hyperplasia, the latter not being FDG avid. ${ }^{53}$ Before routine utilization of $\mathrm{PET} / \mathrm{CT}$ can be recommended, further investigations of the effectiveness of PET/CT in FLC are warranted.

\section{Pathology}

While cross-sectional imaging can strongly suggest FLC, confirmation of the diagnosis can only be achieved with the use of a biopsy. While a needle biopsy is often obtained, a definitive diagnosis can be difficult to confirm by fineneedle biopsy, and occasionally, additional tissue (eg, core biopsy) is required for accurate diagnosis. It is important to note, however, that biopsy is typically not necessary - nor recommended - if the lesion is highly suspicious for FLC on cross-sectional imaging and resection is feasible. Under these circumstances, rather than biopsy, surgery should be recommended. Rather, biopsy should more commonly be reserved for those circumstances of true diagnostic uncertainty or when the lesion is not amenable to resection and the tissue is required to direct other nonsurgical therapy.

On pathology, FLC tumors tend to be large, yellow/tan, hypervascular, well-circumscribed masses with areas of necrosis in otherwise normal liver parenchyma. Up to $75 \%$ of tumors may have a central stellate scar and prominent fibrous tissue. ${ }^{55}$ Microscopically, FLC is characterized by large polygonal or spindle-shaped tumor cells with deeply eosinophilic cytoplasm due to abundant mitochondria and prominent nuclei arranged in cords surrounded by lamellated
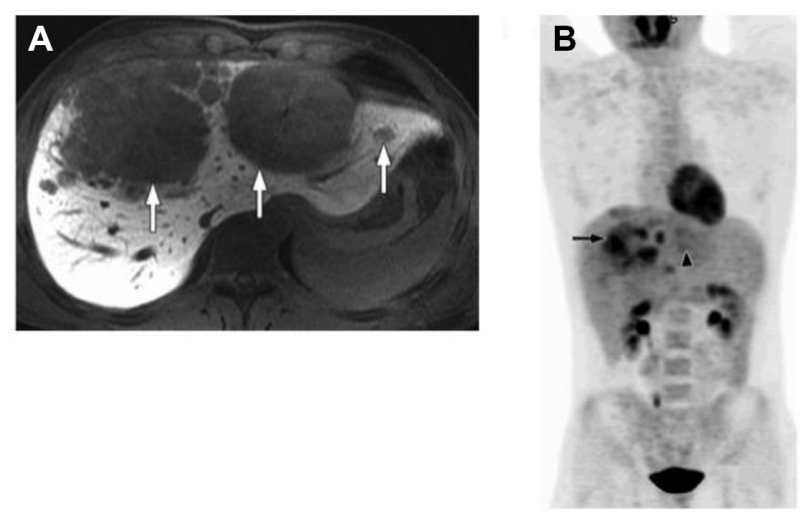

Figure I A 22-year-old man with fibrolamellar HCC.

Notes: (A) Axial contrast-enhanced (gadoxetate disodium, Eovist, Bayer HealthCare), TI-weighted MR image obtained 20 minutes after contrast administration shows large multifocal fibrolamellar hepatocellular carcinoma with satellite lesions (arrows). (B) Coronal maximum-intensity projection image of ${ }^{18} \mathrm{~F}-\mathrm{FDG}$ PET/CT shows that tumor has heterogeneous FDG avidity, with some lesions more FDG avid (arrow) than others (arrowhead). Reprinted with permission from the American Journal of Roentgenology. ${ }^{50}$

Abbreviations: HCC, hepatocellular carcinoma; MR, magnetic resonance; PET, positron emission tomography; CT, computed tomography; FDG, fludeoxyglucose. collagen fibers (Figure 2)., ${ }^{3,56}$ In fact, the average size of FLC tumors cells is roughly three times larger than normal hepatocytes and 1.6 times larger than HCC tumor cells. ${ }^{57}$ Round- to oval-shaped cytoplasmic pale bodies lacking a nucleus and intracytoplasmic hyaline droplets are also seen on microscopy but are not required for diagnosis. ${ }^{58}$ Generally, there is no cirrhosis in the surrounding liver parenchyma; however, there may be nonspecific inflammation suggested by the presence of mononuclear cells and lymphocytes. ${ }^{56}$ Electron microscopy often demonstrates an increase in the number of mitochondria - a pathological feature specific to FLC. ${ }^{3}$

Immunohistochemical staining of FLC has some similarities to $\mathrm{HCC}$, including staining positive for hepatocyte paraffin 1. However, unlike HCC, FLC often stains strongly for CK7 and epithelial membrane antigen, which are characteristic of biliary differentiation as well as markers of hepatic differentiation (CK19 and EpCAM). ${ }^{51,59}$ In addition, unlike most HCC, FLC stains negative for alpha fetoprotein. ${ }^{51,59}$ Furthermore, FLC tends to express CD133 and CD44 markers that are associated with stem cells. ${ }^{60} \mathrm{FLC}$ also stains more often and more diffusely for epithelial growth factor receptor and transforming growth factor beta than classic HCC. ${ }^{59,61}$ Transforming growth factor beta has been shown to be a
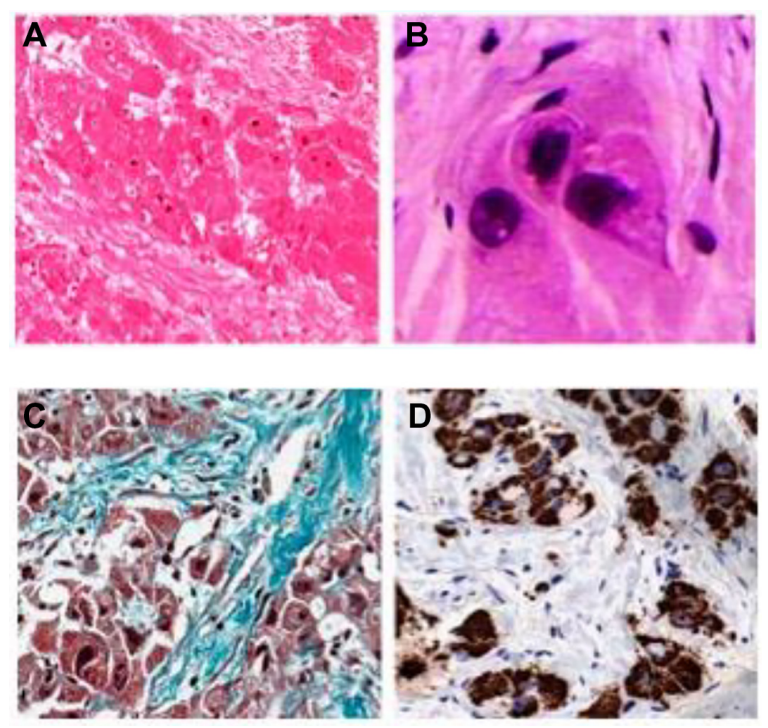

Figure 2 Typical histological features of FLHCC.

Notes: Hematoxylin and eosin staining of tumor tissues (A and $\mathbf{B}$ ) from a patient with FLHCC. Large tumor cells are filled with eosinophilic granular cytoplasm and contain a large vesicular nucleus with a macronucleolus. They are arranged in trabeculae separated by abundant fibrous bands. (A) Low magnification; (B) high magnification. By Masson's trichrome stain (C), dense fibrous bands (greenish blue) between nests of tumor cells are clearly visible. By immunohistochemical staining (D), tumor cells are strongly positive for Hepar, indicating the hepatocellular origin of tumor cells. Original magnifications are $\times 200$ (A, C and D) and $\times 400$ (B). Reprinted by permission from Macmillan Publishers Ltd: The American Journal of Gastroenterology. Liu S, Chan KW, Wang B, Qiao L. Fibrolamellar hepatocellular carcinoma. Am J Gastroenterol. 2009; 104(I0):2617-2624. Copyright (C) 2009.56

Abbreviation: FLHCC, fibrolamellar hepatocellular carcinoma. 
profibrotic factor that may account for the lamellar pattern characteristic of FLC tumors on pathology. ${ }^{59,61}$

In addition to pathologic evaluation and immunohistochemical staining, there are genetic differences discovered recently which distinguish FLC from normal liver parenchyma and HCC. A $400 \mathrm{~kb}$ deletion in chromosome 19 seen in $100 \%$ of the FLC tumors tested by Honeyman et al results in a functional DNAJB1-PRKACA chimeric transcript, which further defines FLC as a unique entity. ${ }^{62,63}$

\section{Treatment}

\section{Surgical resection}

When feasible, surgery is the cornerstone therapeutic modality for patients with FLC as it represents the only potentially curative option. Complete surgical resection of the FLC tumor with negative margins along with an adequate lymph node dissection is the ideal treatment. In a systematic review by Mavros et al, the authors analyzed 575 patients with FLC. ${ }^{64}$ The authors noted that patients who underwent resection of FLC had a 5-year survival of 70\% compared with $0 \%$ among those patients who did not undergo surgical resection. ${ }^{64}$ The average size of the FLC tumor resected was between $9 \mathrm{~cm}$ and $13 \mathrm{~cm} .{ }^{6,50,65}$ In a separate study, Stipa et al reported on 28 resected FLC patients and noted that $75 \%$ of the patients who underwent surgery for FLC required either a hemi-hepatectomy or an extended hepatectomy. ${ }^{6}$ As the surgeries are often complex, a complete ( $\mathrm{R} 0$ ) resection is not always possible, but it is important for survival. In a study by Darcy et al, which looked at 21 patients who underwent resection for FLC at a highly specialized cancer center, a complete (R0) resection was achieved in $17(80.9 \%)$ patients, an R1 in two patients (9.5\%), and an $\mathrm{R} 2$ in two patients $(9.5 \%){ }^{65}$ The overall 5-year survival in this cohort was $42.6 \%$ (95\% confidence interval, 20-65.2), while the 5-year overall survival of those who underwent complete resection was $51.6 \%$. Improved long-term overall survival was associated with $\mathrm{R} 0$ resection $(P=0.003) .{ }^{65}$ In addition to $\mathrm{R} 0$ resection, regional lymph node dissection is warranted due to the high incidence of lymph node metastasis and regional recurrence in patients with nodal disease. .,66,67 $^{6}$

Several factors are associated with a better prognosis following surgery including younger age at diagnosis, earlier tumor stage at diagnosis, as well as absence of large vessel invasion or thrombosis., ${ }^{5,12}$ Factors associated with a particularly poor prognosis include lymph node metastasis, multiple tumors, metastatic disease at presentation, and vascular invasion. ${ }^{6,13,65,68}$ There have been conflicting data regarding sex as a prognostic factor, as studies have variably reported female sex to be both a favorable and adverse factor associated with long-term survival (Table 1). 5,68,69
Table I Prognostic factors in fibrolamellar carcinoma

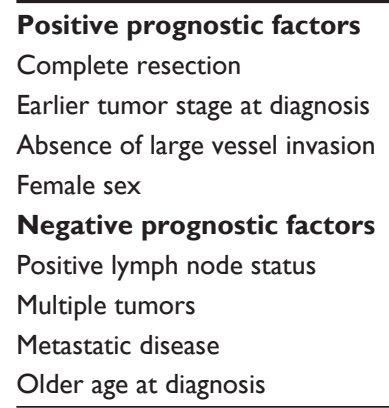

Prognosis following resection of FLC has also been suggested to be better than typical HCC (Figure 3)., ${ }^{3,5-7,58,70}$ There are several factors that may contribute to the better prognosis of FLC patients, including that FLC patients are typically younger and healthier. In addition, FLC patients have normal underlying liver parenchyma, which may allow for more aggressive resections and decrease the risk of de novo future disease. As noted, the ability to perform complete resection has been reported to be one of the most important and well described prognostic factors for FLC. $5,6,65,68,71$

Despite a generally good long-term prognosis, recurrence following resection of FLC is relatively common with rates ranging from $33 \%$ to $100 \%$ and a median recurrence-free survival of 20-48 months. ${ }^{6,72}$ For example, in a small series of 28 patients who underwent resection of FLC, Stipa et al reported a 5-year recurrence-free survival of only $18 \%$ with an overall recurrence rate of $60 \%{ }^{6}$ The most common sites of recurrence include lymph nodes, liver, peritoneum, lungs, and bone. ${ }^{73}$ Due to the

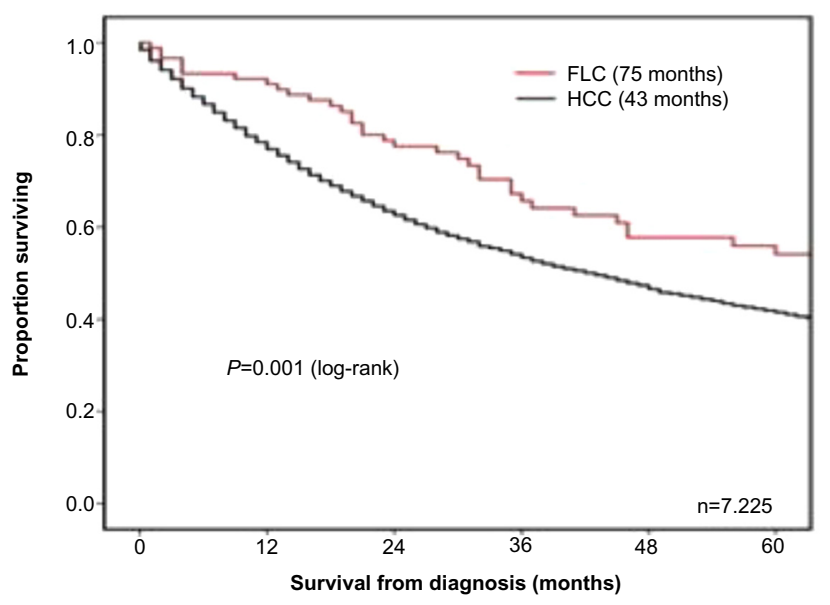

Figure 3 Overall survival of patients with FLC or HCC managed with a liverdirected procedure from the time of diagnosis in SEER from 1986 to 2008.

Notes: FLC ( $n=90$; median survival: 75 months). HCC ( $n=7,135$; median survival: 43 months). $P=0.001$. Reprinted from Journal of the American College of Surgeons, 2I8(2), Mayo SC, Mavros MN, Nathan H, et al, Treatment and prognosis of patients with fibrolamellar hepatocellular carcinoma: a national perspective, 196-205, Copyright (C) 2014, with permission from Elsevier. ${ }^{70}$

Abbreviations: FLC, fibrolamellar carcinoma; HCC, hepatocellular carcinoma; SEER, Surveillance, Epidemiology, and End Results Program. 
high recurrence associated with FLC, diligent postoperative surveillance is indicated. For example, Maniaci et al have proposed an intensive surveillance protocol following surgery that includes $\mathrm{CT}$ and serum vitamin B12-binding protein levels every 3-6 months for the first 2-3 years postoperatively. ${ }^{54}$ In cases where serum vitamin B12-binding protein is elevated and CT scan is negative, the authors recommend PET/CT.${ }^{54}$ While this or other protocols have not been vigorously studied - and therefore cannot be endorsed - the data collectively suggest that close surveillance is warranted. If recurrence is detected, depending on the site and number of recurrent lesions, repeat surgical resection should be considered, as other treatment options are not effective. For example, Maniaci et al reported on ten patients with FLC treated with resection followed by close surveillance and re-excision, systemic chemotherapy, as well as radiotherapy for any relapses. This study showed a median overall survival of 9.3 years $(95 \%$ confidence interval, 3.0-18.5) with two patients showing at least partial response to cisplatin and fluorouracil. ${ }^{54}$

For patients with FLC who present with unresectable disease, liver transplantation should be considered as 3-year survival following $75 \%-80 \%$ transplantation approaches. ${ }^{74}$ While transplantation may be used in cases of FLR, it is much more commonly indicated for HCC than FLC. ${ }^{70}$ This is likely due to the fact HCC is more common than FLC as well as the fact that regional lymph node metastasis (a relative contraindication to transplant) is more common in FLC $(42.2 \%)$ compared with HCC (22.2\%). ${ }^{70}$

\section{Systemic therapy}

FLC is not typically responsive to chemotherapy. While there is no consensus regarding the ideal chemotherapeutic regimen for FLC, platinum-based chemotherapy regimens, as well as combination regimens including interferon alpha- $2 b$ have been used with some success. ${ }^{54,75,76}$ In a Phase II trial, Patt et al reported a complete or partial response in five out of eight patients treated with a combination therapy of fluorouracil and recombinant interferon alpha- $2 b .^{75,76}$ Recent case reports have described the use of gemcitabine/oxaliplatin and 5-FU/ folinic acid/oxaliplatin with some success. ${ }^{77-79}$

In addition to systemic chemotherapy, recent research has focused on taking advantage of the new understanding of the pathogenesis and molecular genetics of FLC. For example, one current multi-institutional, randomized controlled trial is evaluating mTOR inhibition in combination with estrogen suppression in the treatment of FLC. ${ }^{80}$ In addition, FLC has been shown to express increased levels of epithelial growth factor receptor as well as transforming growth factor beta. ${ }^{81}$ Thus, these two factors are potential targets in the future treatment of FLC.

\section{Locoregional therapy}

As FLC is not typically responsive to systemic chemotherapy, locoregional therapies have been considered. While not well studied, radiation therapy has been used to treat recurrent FLC in a few small case series. In one case report, Peacock et al demonstrated an $85 \%$ decrease in tumor volume of FLC metastases using 40 Gy in ten fractions over a 13-day time period. ${ }^{82}$ External beam radiation therapy was delivered as $21 \mathrm{~Gy}$ to the involved field in seven fractions over 10 days for most patients. In a separate retrospective study of ten patients with nonresectable metastatic disease who were treated with external beam radiation in addition to chemotherapy, three patients had objective partial responses by volumetric analysis, six patients had stabilization of their tumor volume, and one patient had early progression. ${ }^{83}$ While regional liverdirected therapies (eg, chemoembolization, yttrium 90, ablation) have been well described for $\mathrm{HCC}$, their use in FLC remains poorly defined.

\section{Conclusion}

FLC has a distinct epidemiology, radiographic appearance, as well as pathologic characteristic than HCC. Most often, patients who present with FLC have an absence of common risk factors seen in classic HCC. While physical findings are often not helpful, cross-sectional imaging with CT or MRI will typically display features highly suggestive of FLC. For patients with resectable disease, surgical resection with lymphadenectomy is the recommended treatment. The long-term prognosis for patients with resected FLC is good; however, many patients will experience a recurrence. A subset of patients who recur may be candidates for surgery. For patients who present with initially unresectable disease or develop an unresectable recurrence, other therapeutic options including systemic or locoregional therapy should be considered. Unfortunately, nonsurgical options for patients with FLC remain limited, and future research is needed to identify better multimodality therapies.

\section{Disclosure}

The authors report no conflicts of interest in this work.

\section{References}

1. National Cancer Institute D SRP, Cancer Statistics Branch. Surveillance, Epidemiology, and End Results (SEER) Program; 2015. Available from: http://www.seer.cancer.gov.

2. Edmondson HA. Differential diagnosis of tumors and tumor-like lesions of liver in infancy and childhood. AMA J Dis Child. 1956;91(2):168-186.

3. Craig JR, Peters RL, Edmondson HA, Omata M. Fibrolamellar carcinoma of the liver: a tumor of adolescents and young adults with distinctive clinico-pathologic features. Cancer. 1980;46(2):372-379. 
4. Bosman FT, World Health Organization; International Agency for Research on Cancer. WHO Classification of Tumours of the Digestive System. 4th ed. Lyon: International Agency for Research on Cancer; 2010.

5. El-Serag HB, Davila JA. Is fibrolamellar carcinoma different from hepatocellular carcinoma? A US population-based study. Hepatology. 2004;39(3):798-803.

6. Stipa F, Yoon SS, Liau KH, et al. Outcome of patients with fibrolamellar hepatocellular carcinoma. Cancer. 2006;106(6):1331-1338.

7. Berman MM, Libbey NP, Foster JH. Hepatocellular carcinoma. Polygonal cell type with fibrous stroma - an atypical variant with a favorable prognosis. Cancer. 1980;46(6):1448-1455.

8. Arista-Nasr J, Gutierrez-Villalobos L, Nuncio J, Maldonaldo H, Bornstein-Quevedo L. Fibrolamellar hepatocellular carcinoma in Mexican patients. Pathol Oncol Res. 2002;8(2):133-137.

9. Bismuth H, Chiche L, Castaing D. Surgical treatment of hepatocellular carcinomas in noncirrhotic liver: experience with 68 liver resections. World J Surg. 1995;19(1):35-41.

10. Hemming AW, Langer B, Sheiner P, Greig PD, Taylor BR. Aggressive surgical management of fibrolamellar hepatocellular carcinoma. J Gastrointest Surg. 1997;1(4):342-346.

11. Moore SW, Davidson A, Hadley GP, et al. Malignant liver tumors in South African children: a national audit. World J Surg. 2008;32(7): 1389-1395.

12. Moreno-Luna LE, Arrieta O, García-Leiva J, et al. Clinical and pathologic factors associated with survival in young adult patients with fibrolamellar hepatocarcinoma. BMC Cancer. 2005;5:142.

13. Pinna AD, Iwatsuki S, Lee RG, et al. Treatment of fibrolamellar hepatoma with subtotal hepatectomy or transplantation. Hepatology. 1997;26(4):877-883.

14. Stevens WR, Johnson CD, Stephens DH, Nagorney DM. Fibrolamellar hepatocellular carcinoma: stage at presentation and results of aggressive surgical management. Am J Roentgenol. 1995;164(5):1153-1158.

15. Teitelbaum DH, Tuttle S, Carey LC, Clausen KP. Fibrolamellar carcinoma of the liver. Review of three cases and the presentation of a characteristic set of tumor markers defining this tumor. Ann Surg. 1985;202(1):36-41.

16. Kaczynski J, Gustavsson B, Hansson G, Wallerstedt S. Fibrolamellar hepatic carcinoma in an area with a low incidence of primary liver cancer: a retrospective study. Eur J Surg. 1996;162(5):367-371.

17. Sooklim K, Sriplung H, Piratvisuth T. Histologic subtypes of hepatocellular carcinoma in the southern Thai population. Asian Pac J Cancer Prev. 2003;4(4):302-306.

18. Al-Matham K, Alabed I, Zaidi SZ, Qushmaq KA. Cold agglutinin disease in fibrolamellar hepatocellular carcinoma: a rare association with a rare cancer variant. Ann Saudi Med. 2011;31(2):197-200.

19. Dadke D, Jaganath P, Krishnamurthy S, Chiplunkar S. The detection of HBV antigens and HBx-transcripts in an Indian fibrolamellar carcinoma patient: a case study. Liver. 2002;22(1):87-91.

20. Haratake J, Horie A, Lee SD, Huh MH. Fibrolamellar carcinoma of the liver in a middle-aged Korean man. J UOEH. 1990;12(3):349-354.

21. Hoshino H, Katada N, Nishimura D, et al. Case report: fibrolamellar hepatocellular carcinoma in a Japanese woman: a case report and review of Japanese cases. J Gastroenterol Hepatol. 1996;11(6):551-555.

22. Paradinas FJ, Melia WM, Wilkinson ML, et al. High serum vitamin B12 binding capacity as a marker of the fibrolamellar variant of hepatocellular carcinoma. Br Med J. 1982;285(6345):840-842.

23. Thirabanjasak D, Sosothikul D, MahayosnondA, Thorner PS. Fibrolamellar carcinoma presenting as a pancreatic mass: case report and review of the literature. J Pediatr Hematol Oncol. 2009;31(5):370-372.

24. Yen JB, Chang KW. Fibrolamellar hepatocellular carcinoma- report of a case. Chang Gung Med J. 2009;32(3):336-339.

25. Yuan CY, Yuan CC, Shiou GF, Tseng CH, Yau MT. Fibrolamellar variant of hepatocellular carcinoma - report of a Chinese patient. Hepatogastroenterology. 1995;42(2):182-184.

26. Vaideeswar P, Pandit MJ, Deshpande JR, Sivaraman A, Vora IM. Fibrolamellar carcinoma of the liver - an unusual presentation. J Postgrad Med. 1993;39(3):159-161.
27. Albaugh JS, Keeffe EB, Krippaehne WW. Recurrent obstructive jaundice caused by fibrolamellar hepatocellular carcinoma. Dig Dis Sci. 1984;29(8):762-767.

28. Eckstein RP, Bambach CP, Stiel D, Roche J, Goodman BN. Fibrolamellar carcinoma as a cause of bile duct obstruction. Pathology. 1988;20(4): 326-331.

29. Soyer P, Roche A, Levesque M. Fibrolamellar hepatocellular carcinoma presenting with obstructive jaundice. A report of two cases. Eur $J$ Radiol. 1991;13(3):196-198.

30. McCloskey JJ, Germain-Lee EL, Perman JA, Plotnick LP, Janoski AH. Gynecomastia as a presenting sign of fibrolamellar carcinoma of the liver. Pediatrics. 1988;82(3):379-382.

31. Athanasakis E, Mouloudi E, Prinianakis G, Kostaki M, Tzardi M, Georgopoulos D. Metastatic liver disease and fulminant hepatic failure: presentation of a case and review of the literature. Eur J Gastroenterol Hepatol. 2003;15(11):1235-1240.

32. Myszor MF, Record CO. Primary and secondary malignant disease of the liver and fulminant hepatic failure. J Clin Gastroenterol. 1990; 12(4):441-446.

33. Soreide O, Czerniak A, Bradpiece H, Bloom S, Blumgart L. Characteristics of fibrolamellar hepatocellular carcinoma. A study of nine cases and a review of the literature. Am J Surg. 1986;151(4):518-523.

34. Marrannes J, Gryspeerdt S, Haspeslagh M, van Holsbeeck B, Baekelandt M, Lefere P. Fibrolamellar hepatocellular carcinoma in a 65-year-old woman: CT features. JBR-BTR. 2005;88(5):237-240.

35. Sethi S, Tageja N, Singh J, et al. Hyperammonemic encephalopathy: a rare presentation of fibrolamellar hepatocellular carcinoma. Am J Med Sci. 2009;338(6):522-524.

36. Mansouri D, Van Nhieu JT, Couanet D, et al. Fibrolamellar hepatocellular carcinoma: a case report with cytological features in a sixteenyear-old girl. Diagn Cytopathol. 2006;34(8):568-571.

37. Tanaka J, Baba N, Arii S, et al. Typical fibrolamellar hepatocellular carcinoma in Japanese patients: report of two cases. Surg Today. 1994;24(5):459-463.

38. Gupta P, Dhar S, Strickland NH. Fibrolamellar carcinoma: an unusual clinico-radiological presentation. Eur J Radiol. 1999;32(2):119-123.

39. Tangkijvanich P, Thong-Ngam D, Kullavanijaya P, Suwangool P. Fibrolamellar hepatocellular carcinoma in a Thai man who presented with hypoglycemia: case report and review of literature. J Med Assoc Thai. 2000;83(7):809-816.

40. Dahan MH, Kastell P. Fibrolamellar hepatic carcinoma with a presentation similar to that of septic pregnancy. A case report. J Reprod Med. 2002;47(1):47-49.

41. Torbenson M. Fibrolamellar carcinoma: 2012 update. Scientifica. 2012;2012:743790.

42. Lloreta J, Vadell C, Fabregat X, Serrano S. Fibrolamellar hepatic tumor with neurosecretory features and systemic deposition of AA amyloid. Ultrastruct Pathol. 1994;18(1-2):287-292.

43. Ward SC, Huang J, Tickoo SK, Thung SN, Ladanyi M, Klimstra DS. Fibrolamellar carcinoma of the liver exhibits immunohistochemical evidence of both hepatocyte and bile duct differentiation. Mod Pathol. 2010;23(9):1180-1190.

44. Frémont $\mathrm{S}$, Champigneulle $\mathrm{B}$, Gérard $\mathrm{P}$, et al. Blood transcobalamin levels in malignant hepatoma. Tumour Biol. 1991;12(6):353-359.

45. Lildballe DL, Nguyen KQ, Poulsen SS, Nielsen HO, Nexo E. Haptocorrin as marker of disease progression in fibrolamellar hepatocellular carcinoma. Eur J Surg Oncol. 2011;37(1):72-79.

46. Wheeler K, Pritchard J, Luck W, Rossiter M. Transcobalamin I as a "marker" for fibrolamellar hepatoma. Med Pediatr Oncol. 1986;14(4): 227-229.

47. Kanai T, Takabayashi T, Kawano Y, Kuramochi S, Miyazawa N. A case of postoperative recurrence of fibrolamellar hepatocellular carcinoma with increased vitamin B12 binding capacity in a young Japanese female. Jpn J Clin Oncol. 2004;34(6):346-351.

48. Collier NA, Weinbren K, Bloom SR, Lee YC, Hodgson HJ, Blumgart LH. Neurotensin secretion by fibrolamellar carcinoma of the liver. Lancet. 1984;1(8376):538-540. 
49. Friedman AC, Lichtenstein JE, Goodman Z, Fishman EK, Siegelman SS, Dachman AH. Fibrolamellar hepatocellular carcinoma. Radiology. 1985;157(3):583-587.

50. Ganeshan D, Szklaruk J, Kundra V, Kaseb A, Rashid A, Elsayes KM. Imaging features of fibrolamellar hepatocellular carcinoma. Am J Roentgenol. 2014;202(3):544-552.

51. Ichikawa T, Federle MP, Grazioli L, Madariaga J, Nalesnik M, Marsh W. Fibrolamellar hepatocellular carcinoma: imaging and pathologic findings in 31 recent cases. Radiology. 1999;213(2):352-361.

52. Blachar A, Federle MP, Ferris JV, et al. Radiologists' performance in the diagnosis of liver tumors with central scars by using specific CT criteria. Radiology. 2002;223(2):532-539.

53. Liu S, Wah Chan K, Tong J, Wang Y, Wang B, Qiao L. PET-CT scan is a valuable modality in the diagnosis of fibrolamellar hepatocellular carcinoma: a case report and a summary of recent literature. QJM. 2011;104(6):477-483.

54. Maniaci V, Davidson BR, Rolles K, et al. Fibrolamellar hepatocellular carcinoma: prolonged survival with multimodality therapy. Eur J Surg Oncol. 2009;35(6):617-621.

55. Ichikawa T, Federle MP, Grazioli L, Marsh W. Fibrolamellar hepatocellular carcinoma: pre- and posttherapy evaluation with CT and MR imaging. Radiology. 2000;217(1):145-151.

56. Liu S, Chan KW, Wang B, Qiao L. Fibrolamellar hepatocellular carcinoma. Am J Gastroenterol. 2009;104(10):2617-2624. [quiz 2625].

57. Perez-Guillermo M, Masgrau NA, Garcia-Solano J, Sola-Perez J, de Agustin y de Agustin P. Cytologic aspect of fibrolamellar hepatocellular carcinoma in fine-needle aspirates. Diagn Cytopathol. 1999;21(3):180-187.

58. Berman MA, Burnham JA, Sheahan DG. Fibrolamellar carcinoma of the liver: an immunohistochemical study of nineteen cases and a review of the literature. Hum Pathol. 1988;19(7):784-794.

59. Ward SC, Waxman S. Fibrolamellar carcinoma: a review with focus on genetics and comparison to other malignant primary liver tumors. Semin Liver Dis. 2011;31(1):61-70.

60. Zenali MJ, Tan D, Li W, Dhingra S, Brown RE. Stemness characteristics of fibrolamellar hepatocellular carcinoma: immunohistochemical analysis with comparisons to conventional hepatocellular carcinoma. Ann Clin Lab Sci. 2010;40(2):126-134.

61. Orsatti G, Hytiroglou P, Thung SN, Ishak KG, Paronetto F. Lamellar fibrosis in the fibrolamellar variant of hepatocellular carcinoma: a role for transforming growth factor beta. Liver. 1997;17(3):152-156.

62. Honeyman JN, Simon EP, Robine N, et al. Detection of a recurrent DNAJB1-PRKACA chimeric transcript in fibrolamellar hepatocellular carcinoma. Science. 2014;343(6174):1010-1014.

63. Darcy DG, Chiaroni-Clarke R, Murphy JM, et al. The genomic landscape of fibrolamellar hepatocellular carcinoma: whole genome sequencing of ten patients. Oncotarget. 2015;6(2):755-770.

64. Mavros MN, Mayo SC, Hyder O, Pawlik TM. A systematic review: treatment and prognosis of patients with fibrolamellar hepatocellular carcinoma. J Am Coll Surg. 2012;215(6):820-830.

65. Darcy DG, MalekMM,Kobos R, Klimstra DS, DeMatteo R, La Quaglia MP. Prognostic factors in fibrolamellar hepatocellular carcinoma in young people. J Pediatr Surg. 2015;50(1):153-156.

66. McAteer JP, Goldin AB, Healey PJ, Gow KW. Hepatocellular carcinoma in children: epidemiology and the impact of regional lymphadenectomy on surgical outcomes. J Pediatr Surg. 2013;48(11):2194-2201.
67. Amini N, Ejaz A, Spolverato G, Maithel SK, Kim Y, Pawlik TM. Management of lymph nodes during resection of hepatocellular carcinoma and intrahepatic cholangiocarcinoma: a systematic review. J Gastrointest Surg. 2014;18(12):2136-2148.

68. Ang CS, Kelley RK, Choti MA, et al. Clinicopathologic characteristics and survival outcomes of patients with fibrolamellar carcinoma: data from the fibrolamellar carcinoma consortium. Gastrointest Cancer Res. 2013;6(1):3-9.

69. Kaseb AO, Shama M, Sahin IH, et al. Prognostic indicators and treatment outcome in 94 cases of fibrolamellar hepatocellular carcinoma. Oncology. 2013;85(4):197-203.

70. Mayo SC, Mavros MN, Nathan H, et al. Treatment and prognosis of patients with fibrolamellar hepatocellular carcinoma: a national perspective. J Am Coll Surg. 2014;218(2):196-205.

71. Katzenstein HM, Krailo MD, Malogolowkin MH, et al. Fibrolamellar hepatocellular carcinoma in children and adolescents. Cancer. 2003; 97(8):2006-2012.

72. Groeschl RT, Miura JT, Wong RK, et al. Multi-institutional analysis of recurrence and survival after hepatectomy for fibrolamellar carcinoma. J Surg Oncol. 2014;110(4):412-415.

73. Wojcicki M, Lubikowski J, Post M, Chmurowicz T, WiechowskaKozlowska A, Krawczyk M. Aggressive surgical management of recurrent lymph node and pancreatic head metastases of resected fibrolamellar hepatocellular carcinoma: a case report. JOP. 2012;13(5): 529-532.

74. El-Gazzaz G, Wong W, El-Hadary MK, et al. Outcome of liver resection and transplantation for fibrolamellar hepatocellular carcinoma. Trans Int. 2000;13(Suppl 1):S406-S409.

75. Bower M, Newlands ES, Habib N. Fibrolamellar hepatocellular carcinoma responsive to platinum-based combination chemotherapy. Clin Oncol. 1996;8(5):331-333.

76. Patt YZ, Hassan MM, Lozano RD, et al. Phase II trial of systemic continuous fluorouracil and subcutaneous recombinant interferon Alfa-2b for treatment of hepatocellular carcinoma. J Clin Oncol. 2003; 21(3):421-427.

77. Fonseca GM, Varella AD, Coelho FF, Abe ES, Dumarco RB, Herman P. Downstaging and resection after neoadjuvant therapy for fibrolamellar hepatocellular carcinoma. World J Gastrointest Surg. 2014;6(6):107-111.

78. Fakih M. A case of fibrolamellar cancer with a palliative response and minor radiographic regression with erlotinib and bevacizumab combination therapy. Am J Ther. 2014;21(6):e207-e210.

79. Gras P, Truant S, Boige V, et al. Prolonged Complete Response after GEMOX Chemotherapy in a Patient with Advanced Fibrolamellar Hepatocellular Carcinoma. Case Rep Oncol. 2012;5(1):169-172.

80. Riehle KJ, Yeh MM, Yu JJ, et al. mTORC1 and FGFR1 signaling in fibrolamellar hepatocellular carcinoma. Mod Pathol. 2015;28(1): 103-110.

81. Buckley AF, Burgart LJ, Kakar S. Epidermal growth factor receptor expression and gene copy number in fibrolamellar hepatocellular carcinoma. Hum Pathol. 2006;37(4):410-414.

82. Peacock JG, Call JA, Olivier KR. Radiotherapy for metastatic fibrolamellar hepatocellular carcinoma. Rare Tumors. 2013;5(3):e28.

83. Epstein BE, Pajak TF, Haulk TL, Herpst JM, Order SE, Abrams RA. Metastatic nonresectable fibrolamellar hepatoma: prognostic features and natural history. Am J Clin Oncol. 1999;22(1):22-28.

Journal of Hepatocellular Carcinoma

\section{Publish your work in this journal}

The Journal of Hepatocellular Carcinoma is an international, peerreviewed, open access journal that offers a platform for the dissemination and study of clinical, translational and basic research findings in this rapidly developing field. Development in areas including, but not limited to, epidemiology, vaccination, hepatitis therapy, pathology and

molecular tumor classification and prognostication are all considered for publication. The manuscript management system is completely online and includes a very quick and fair peer-review system, which is all easy to use. Visit http://www.dovepress.com/testimonialsphp to read real quotes from published authors. 